\title{
Treatment of Pelvic Ring Fractures: Percutaneous Computer Assisted Iliosacral Screwing
}

\author{
Lionel CARRAT ${ }^{a}$, Jerome TONETTI ${ }^{b}$, M.D., Stephane LAVALLEE ${ }^{a}$, Ph.D. \\ Philippe MERLOZ ${ }^{c}$, M.D., Laurence PITTET ${ }^{d}$, M.D. and Jean-Paul \\ CHIROSSEL $^{b}$, M.D. \\ ${ }^{a}$ TIMC Laboratory, Faculté de Médecine, I.A.B., 38706 La Tronche (France) \\ ${ }^{b}$ Anatomy Laboratory, Pr Chirossel, University Joseph Fourier 38043 Grenoble \\ ${ }^{c}$ Orthopaedic Department, CHU A. Michallon - BP 217 - 38043 Grenoble \\ ${ }^{d}$ Radiology Department, CHU A. Michallon - BP 217 - 38043 Grenoble
}

e-mail : Lionel.Carrat@imag.fr

\begin{abstract}
This paper describes the development and preliminary testing of an image-guided system for the placement of iliosacral screws to stabilize pelvic ring fractures percutaneously, with the aim of decreasing the incidence of surgical complications and increasing the accuracy of screw placement. Pre-operative planning of screw trajectories is performed on a $3 \mathrm{D}$ model of the pelvis constructed from CT scans. During surgery, a $6 \mathrm{D}$ optical localizer is used to track the positions and orientations of an ultrasound probe, a surgical drill and a reference frame fixed to the posterior iliac crest. Registration of the pre-operative model with curves segmented from the ultrasound scans is performed using a surface-based algorithm. The drill tip and axis are displayed relative to the desired screw trajectories in real time. The accuracy of the system has been verified in four cadaver specimens by comparing actual screw trajectories to the desired trajectories and by measuring the distance from each screw to important anatomical landmarks on post-operative CT scans. All screws were considered to be in correct position.
\end{abstract}

\section{Introduction}

Unstable pelvic ring fractures with complete posterior ring disruption (Fig. 1) justify a surgical fixation as described in [1]. The non-operative treatment is not adequate to stabilize the closed reduction in order to prevent hemipelvic ascension or pseudarthrosis of the fracture.

The current technique is open reduction with internal fixation, described in $[2,3,4]$, which consists in introducing 2 screws through the iliosacral joint into the sacral ala and the body of the first sacral vertebra (Fig. 2). The trajectory of the drill must respect the close anatomic elements [5] which are the first sacral nerve root in the S1-S2 foramen, lumbosacral nerve trunc, roots of the cauda equina, iliac vessels and the peritonial cavity. In order to secure the procedure, empirical reference marks must be taken. As a consequence, a large surgical exposure of the sacrum and the posterior part of the fossa iliaca lateral must be done. Complications like blood loss, hematoma and wound infection are not rare 

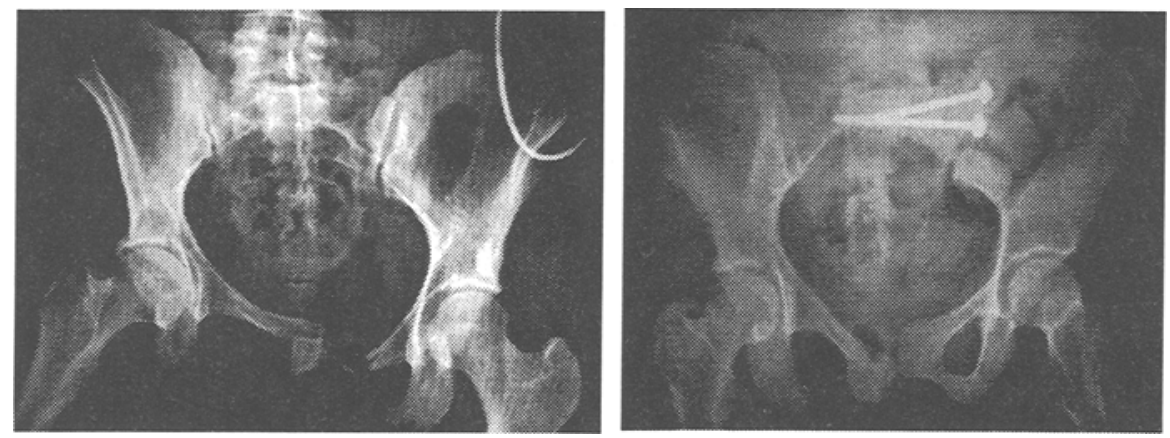

Fig. 1. Unstable pelvic ring fracture with Fig. 2. The screwing technique complete disruption of the posterior arch.

after such exposure because of the perineum's proximity. Percutaneous technique using fluoroscopy or computer tomography, described in $[6,3,7]$, minimize the rate of wound complications. But these techniques require a great experience of the surgeon [5]. Furthermore, digestive gases make the vision difficult.

This article presents a technique using computer assisted guiding method in order to increase accuracy and to decrease the rate of wound complications. Our approach is based on standard computer assisted surgery using pre-operative CT-scan model registered with ultrasound data of the anatomical subject during surgery.

\section{Material and methods}

Our protocol is divided into three steps.

\section{Surgical planning.}

A CT-scan acquisition of nearly 50 images with a $3 \mathrm{~mm}$ slice thickness is performed with a spiral CT GE Hispeed Advantage. The examination field include all the pelvic bone from the top of the iliac crest to the ischiatic process. A semiautomatic segmentation of the bone is performed in order to have a $3 \mathrm{D}$ model of the pelvis. The surgeon defines the optimal placement of the screws described by the entry point, direction, length and diameter. An interactive interface allows the surgeon to compute planes of arbitrary orientation in the volume of CT-scan images. For each pelvis 3 optimal screw positions have been defined on one side of the pelvis (one from supraforaminal S1-S2 superior into the $\mathrm{S} 1$ vertebra's body, one from supraforaminal S1-S2 inferior into the $\mathrm{S} 1$ body and one from infraforaminal S1-S2 into the S2 body). The screw tips are defined on the mid-sagittal line of the sacrum.

\section{Intra-operative registration and tracking.}

During surgery, a 6D optical localizer Optotrak (Northern Digital, Toronto, Canada) (Fig. 3A) locates the position and orientation of infra-red LED's fixed on customized rigid-bodies (TIMC, laboratory) and on a standard ultrasound 


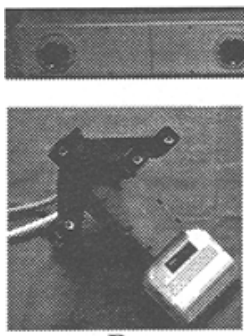

B

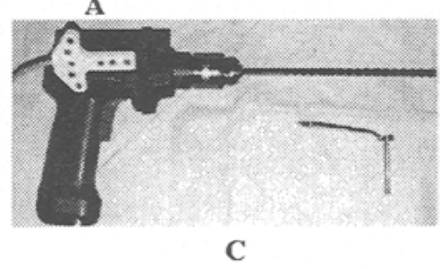

C

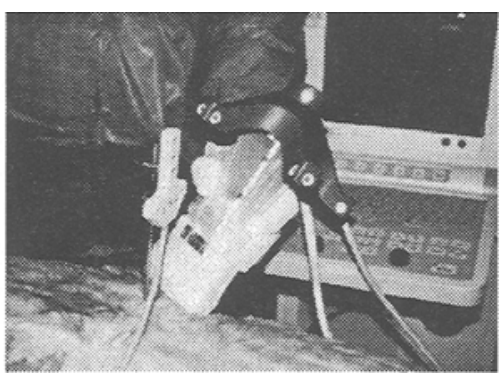

Fig. 3. During surgery, an optical localizer (A) Fig.4. The surgeon makes the ullocates in 3D the position and orientation of trasound acquisition. Thanks to the LED's fixed on rigid-bodies. A standard ultra- localizer, images are located in 3D sound probe (B) and a standard power drill (C) in the intra-operative coordinate are equipped with such rigid-bodies. system.

probe (Fig. 3B) of $7.5 \mathrm{MHz}$ (EUB-405 Hitachi inc, Tokyo, Japan). A rigid-body is also fixed on a standard surgical power drill (Fig. 3C) which will be used for the drilling step. The accuracy of the localizer is better than $0.6 \mathrm{~mm}$ inside a $1 \mathrm{~m}^{3}$ volume. A reference rigid-body is firmly fixed, thanks to a pin, in the posterior iliac crest. It defines the intra-operative reference coordinate system which is used during the whole surgery, whatever the displacements of the bone are. As described in $[8,9]$, we use a standard ultrasound probe to image the bone and soft tissue interface of the iliac ala ipsilateral to the screwing (iliac crest, fossa iliaca lateral, ischiatic processus and posterior side of the sacrum). Nearly $60 \mathrm{ul}-$ trasound images are acquired (Fig. 4). Then, a segmentation procedure enables us to build curves of $2 \mathrm{D}$ points that belong to the bone surface on each image. In most cases, nearly 40 of them are segmented which requires 20 minutes. Several images are not segmented because of their poor quality but acquisition should be better on living humans than on cadavers. Using the $6 \mathrm{D}$ location and the calibration of ultrasound image planes, those curves are converted in clouds of $3 \mathrm{D}$ points referenced in the intra-operative coordinate system. Those clouds of points are registered with the $3 \mathrm{D} \mathrm{CT}$-scan model of the pelvis using surface based registration algorithms of the Stealth software (Sofamor-Danek inc, Memphis, USA)(Fig. 5), thereby avoiding the need to insert fiducial markers into the bones when constructing the pre-operative model. Results of the registration indicate an average rms (root mean squares) error of $0.6 \mathrm{~mm}$ measured on the four pelvis. As a result, the optimal screw positions defined on the CT-scan are known in the intra-operative coordinate system.

\section{Passive drilling guidance.}

The passive guidance process can then be started (Fig. 6). The standard power drill, equipped with a rigid-body, is calibrated. The tip and axis positions can thus be localized and reported in real-time on the computer screen. For each screwing, the optimal trajectory and the real position of the drill are displayed on the screen. The position of the drill is shown on each anatomical view of the 


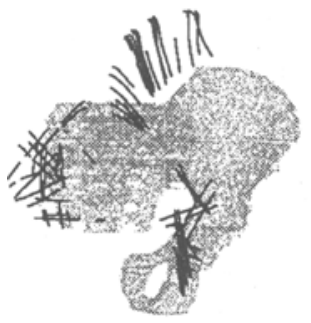

A

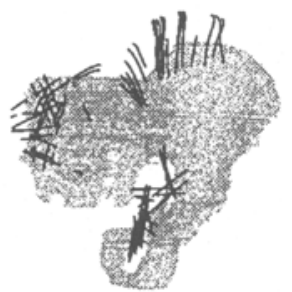

B

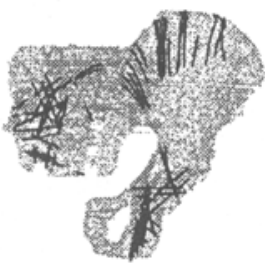

C

Fig. 5. Registration between the CT model (in grey) and the points segmented on ultrasound images of the pelvic (in black). The initial position (A) is the result of a pre-registration made on few data. After a couple of surface-based algoritms iterations, set of data looks like in (B) and the final position is shown in (C).

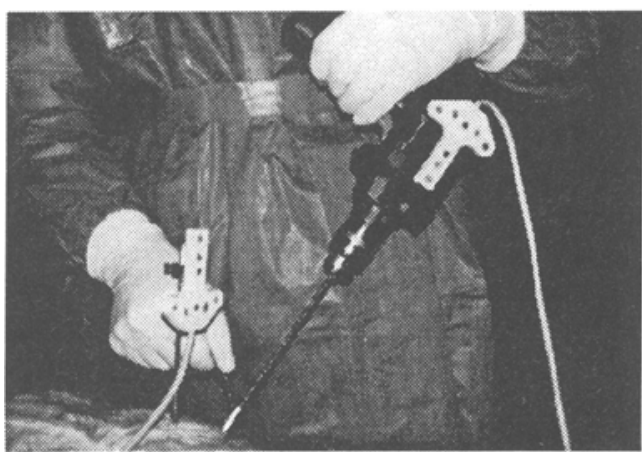

Fig. 6. Passive drilling guidance.

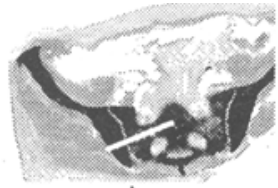

A

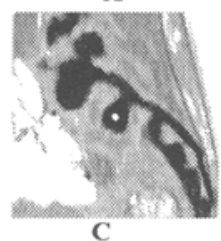

C

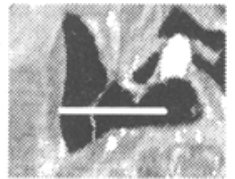

B

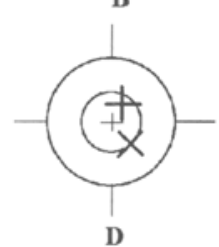

Fig. 7. Real-time monitoring.

pelvic. (Fig. 7A) is the axial view, (Fig. 7B) is the axe view and (Fig. 7C) is the coronal view. On (Fig. 7D) the optimal trajectory is represented by a central cross-hair. The drill tip and axis are projected on a view orthogonal to this trajectory and are represented by two different cross-hairs. The surgeon must superimpose the drill cross-hairs with the central cross-hair. In order to increase the legibility of the visual comparison two circles are displayed. The smaller represents a difference of $2.5 \mathrm{~mm}$, the other represents $5 \mathrm{~mm}$. During the drilling process, the depth penetration of the drill is also displayed and compared with the maximum depth penetration allowed by the CT-scan planning. At the end of the drilling process, the guidance's results are stored. These values are used in order to evaluate the surgeon's accuracy and the drilling process feasibility. We store the distance between real and optimal entry points, target points and depth penetration. Thanks to this assisted guidance, $6.5 \mathrm{~mm}$ diameter screws for cancellous bones (Synthes inc, Etupe, France) are easily inserted in the drilled guide holes. 


\section{Results}

This method has been tested on four cadaver pelvis. We have inserted 3 screws with various trajectories in each.

Ultrasound registration.

With regards to registration, we can compare the results obtained by our ultrasound based method with a standard surface-based registration using 3D points. These points have been digitized directly on the bone surfaces, after dissection at the end of the experiment, by preserving the intra-operative referential. Then, we performed a very stable and accurate registration in order to obtain a gold standard for ultrasound-based registration evaluation. The post-experiment comparison between registration matrix obtained with ultrasound images and with digitized 3D points are measured in terms of rotation and translation. The average errors are $1.47^{\circ}$ in rotation and $2.57 \mathrm{~mm}$ in translation. Those values can be considered as the absolute errors of ultrasound based registration.

\section{Drilling guidance.}

Regarding drilling guidance, the user interface was found to be very practical. However, a minor problem was encountered concerning the mechanical deflection of the drill. As a consequence, the localisation of the tip was inaccurate and errors can be larger than $5 \mathrm{~mm}$. It has been notified for a few specific trajectories. This problem has been solved by using a different mechanical system for guidance (Fig. 8). LED's used for the power drill axis localisation are placed on the drill guide in order to avoid errors introduced by the deflection during the drilling process. Furthermore, we can use the guidance during the screwing process which was not possible before. The depth penetration is still measured thanks to the rigid-body fixed on the power drill and the calibration of the drill tip. At the end of the process, we have saved values which show the accuracy of the guidance. We have measured the distance between optimal and real position

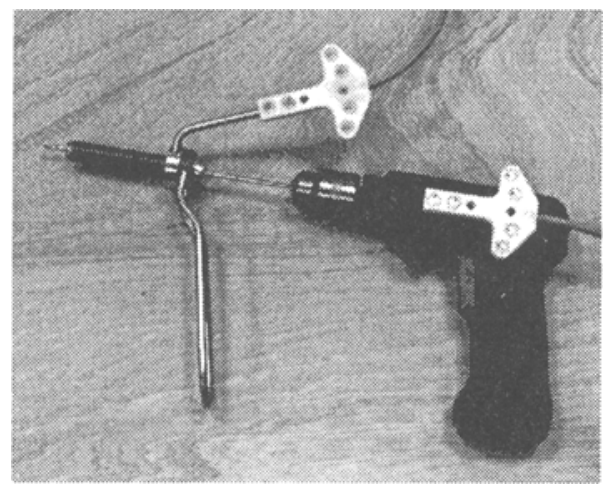

Fig. 8. New materiel used for drilling process. 
of the drill for the entry point, target point and the depth of the tip point for each pelvis. The average errors are $1.9 \mathrm{~mm}$ for entry point, $1.7 \mathrm{~mm}$ for target point and $0.81 \mathrm{~mm}$ for the depth.

\section{Screw positions.}

Screw positions have been checked thanks to a post-operative CT-scan acquisition (Fig. 9). For supraforaminal S1-S2 screws, we have measured the distance from the screw to the anterior cortex of the sacrum, the distance from the screw to the spinal canal or the foramen and the distance from the tip of the screw to the mid-sagittal line of the sacrum (Fig. 9). For infraforaminal screws, we have measured the distance from the screw to the anterior cortex of the sacrum, the distance from the screw to the S2-S3 foramen and the distance from the tip of the screw to the mid-sagittal line of the sacrum. Those values are reported in (Fig. 10). All screws were considered to be in correct position and preserves the close anatomic elements.

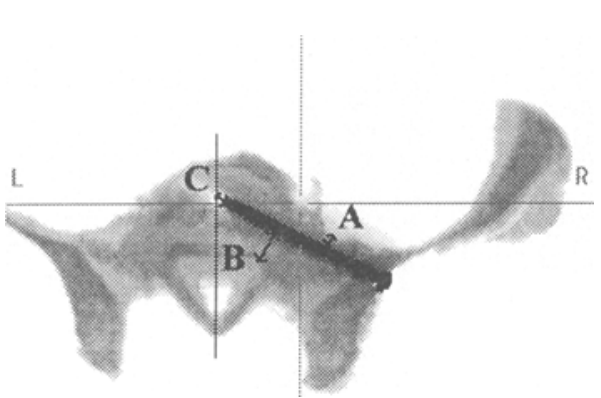

Fig. 9. Exemple of an image taken for CT-verification with method of measurement for screws'position. $A$ is the distance from the screw to the anterior cortex of the sacrum. $B$ is the distance from the screw to the spinal canal or foramen. $C$ is the distance from the tip of the screw to the mid-sagittal line of the sacrum.

\begin{tabular}{|l|l|l|l|l|}
\hline & Screws & A & B & C \\
\hline Pelvis 1 & Suprafor. S1-S2 sup. & 0.5 & 5 & 3 \\
& Suprafor. S1-S2 inf. & 2 & 2 & 1 \\
& Infrafor. S1-S2 & 8 & 0.5 & 1 \\
\hline Pelvis 2 & Suprafor. S1-S2 sup. & 4 & 15 & 1 \\
& Suprafor. S1-S2 inf. & 2 & 14 & 5 \\
& Infrafor. S1-S2 & 9.5 & 4 & 5 \\
\hline Pelvis 3 & Suprafor. S1-S2 sup. & 10 & 1 & 2 \\
& Suprafor. S1-S2 inf. & 3 & 8 & 1 \\
& Infrafor. S1-S2 & 3 & 3 & 2 \\
\hline Pelvis 4 & Suprafor. S1-S2 sup. & 7 & 9 & 1 \\
& Suprafor. S1-S2 inf. & 2 & 6 & 2.5 \\
& Infrafor. S1-S2 & 4 & 2 & 0 \\
\hline \hline Average & & 4.6 & 5.8 & 2 \\
\hline
\end{tabular}

Fig. 10. Measurement of screws placement. $A, B$ and $C$ are in millimeters. $A$ and $B$ values are never equal to 0 . The more the $\mathrm{C}$ value is close to 0 , the better the depth guidance is. (Max. value is $5 \mathrm{~mm}$ ).

\section{Discussion}

Final position of screws depends on the planning, the accuracy of the registration and the drilling process. During the surgical planning, the trajectory is chosen as natural as possible, means into the bony corridor created by the cortical walls of the sacral ala and the sacral vertebra. Guidance process is easier when the tip of the drill is introduced into the bone exactly at the optimal entry point. If so, the drill go deep naturally into the cancellous bone without running against the 
cortical bone. As a consequence, a great accuracy is required at the beginning of the guidance process.

Values measured on CT-scan images for verification were difficult to take, because of artefacts due to the steel of the screws. We have thus measured the narrowest distance between the screw's image and the cortical bone.

Actually, the ultrasound acquisition is performed on the iliac crest, fossa ilaca lateral, ischiatic process and posterior side of the sacrum. The intra-operative reference coordinate system is fixed in the posterior iliac crest. Our current research consists in performing the registration only on the sacrum because accuracy needs to be more important on this part of the pelvis with respect to anatomic elements. Furthermore, it will also avoid the problem of motion at fracture sites relative to pre-operative CT-scan. The intra-operative coordinate system is firmly fixed on the spinal process of the first sacral vertebral of the sacrum. Our approach is to acquire ultrasound images on the sacrum with a smaller probe and to digitize points directly with a pointer. These points are digitized by using short incision used for the fixation of the rigid-body. These data are mixed with $3 \mathrm{D}$ curves obtained by the segmentation of ultrasound images. This method should take advantage of better 3D data which means that the registration should be more accurate and more stable. In addition, it will allow to reduce the reconstruction diameter of the CT-scan because the acquisition is performed only on the sacrum.

The major drawback of our method is concerning the segmentation of ultrasound images. This step takes time (nearly 20 minutes for 40 images) and required experience from the surgeon. Therefore, it is necessary to develop an automated and accurate segmentation. This is our current technical research.

Computer assisted methods have been proposed to perform pelvic surgery [10]. Some authors have already described a material-based registration method in order to insert iliosacral screws [11]. They use a carrier fixed with pins which are implanted in the posterior part of the scarum before the CT-scan examination. With our approach no pelvic instrumentation is performed before the CT-scan. It makes the medical intervention non invasive, pinless and frameless.

\section{Conclusion}

In the framework of computer assisted surgery applied to orthopaedics, this article has presented a percutaneous fixation method for pelvic ring fractures using ultrasound based registration. Our percutaneous technique takes advantage of some previously developed method for spine surgery $[12,13,14]$ because of the decreasing of wound complications. It has been validated successfully on 4 cadaver specimens, which encourages us to start clinical validation. This technique could be applied to fractures with possible closed reduction [7]. But it could also be applied to any other applications of percutaneous orthopaedic surgery. 
Acknowledgements: This research is financially supported by the European project CRIGOS BMH4-CT97-2427.

\section{References}

1. Waddel J.P. Pennal G.F., Tile M. and Garside H. Pelvic disruption : Assessment and classification. Clinical Orthopaedic, (151):12-21, 1980.

2. E. Letournel. Pelvic fractures. Rev. Chir. Orthop., (10):145-148, 1978.

3. JM. Matta and T. Saucedo. Internal fixation of pelvic fracture. Clin. Orthop., (242):83-97, 1989.

4. M. Tile and GF. Pennal. Pelvic disruption : Principe of management. Clin. Orthop., (151):56-64, 1980.

5. Freese J. Templeman D., Schmidt A. and Weisman I. Proximity of iliosacral screws to neurovascular structures after internal fixation. Clinical Orthopaedic, (329):194$198,1996$.

6. Coombs R.J. Jackson W.T. Ebraheim N.A., Russin J.J. and Holiday B. Percutaneous computer-tomography stabilization of pelvic fractures. Prelimnary report. J. Orthop. Trauma., (1):197-204, 1987.

7. M.L. Routt and P.T. Simonian. Closed reduction and percutaneous skeletal fixation of sacral fractures. Clinical Orthopaedic, (329):121-128, 1996.

8. C. Barbe, J. Troccaz, B. Mazier, and S. Lavallee. Using $2.5 \mathrm{D}$ echography in computer assisted spine surgery. In IEEE Engineering in Medicine and Biology Society Proceedings, pages 160-161. IEEE, 1993.

9. R. Mosges and S. Lavallee. Multimodal information for computer-integrated surgery. In R. Taylor, S. Lavallee, G. Burdea, and R. Mosges, editors, Computerintegrated surgery: technology and clinical applications, pages 5-20. MIT Press, Cambridge, MA, 1996.

10. A.L. Jacob. Computer assistance in pelvic and acetabular fractures. In L. Nolte, editor, CAOS'96 (Computer Assisted Orthopaedic Surgery), Bern (CH), 1996. Muller Institute.

11. R. Hu, N. Glossop, D. Steven, and J. Randle. Accuracy of image guided placement of iliosacral lag screws. In J. Troccaz and al, editors, CVRMed-MRCAS'g7 Proc., LNCS Series 1205, pages 593-596. Springer, 1997.

12. S. Lavallee, P. Sautot, J. Troccaz, P. Cinquin, and P. Merloz. Computer Assisted Spine Surgery : a technique for accurate transpedicular screw fixation using CT data and a 3D optical localizer. Journal of Image Guided Surgery, 1(1):65-73, 1995.

13. S. Lavallee, R. Szeliski, and L. Brunie. Anatomy-based registration of 3-D medical images, range images, $\mathrm{X}$-ray projections, 3-D models using Octree-Splines. In R. Taylor, S. Lavallee, G. Burdea, and R. Mosges, editors, Computer Integrated Surgery, pages 115-143. MIT Press, Cambridge, MA, 1996.

14. P. Merloz, J. Tonetti, A. Eid, C. Faure, S. Lavallee, J. Troccaz, P. Sautot, A. Hamadeh, and P. Cinquin. Computer Assisted Spine Surgery. Clinical Orthopaedics and related Research, (337):86-96, 1997. 